\title{
The Discovery of Subatomic Particles : From Atoms to Quarks
}

\author{
by Rochelle Forrester \\ Copyright (C) 2019 Rochelle Forrester \\ All Rights Reserved \\ The moral right of the author has been asserted \\ Anyone may reproduce all or any part of this paper without the permission of the author so long as a full \\ acknowledgement of the source of the reproduced material is made. \\ Second Edition
}

Published 30 September 2019

Preface

This paper was written in order to examine the order of discovery of significant developments in the history of humankind. It is part of my efforts to put the study of social and cultural history and social change on a scientific basis capable of rational analysis and understanding. This has resulted in a hard copy book How Change Happens: A Theory of Philosophy of History, Social Change and Cultural Evolution and a website How Change Happens Rochelle Forrester's Social Change, Cultural Evolution and Philosophy of History website. There are also philosophy of history papers such as The Course of History, The Scientific Study of History, Guttman Scale Analysis and its use to explain Cultural Evolution and Social Change and the Philosophy of History and papers on Academia.edu, Figshare, $\underline{\text { Humanities Common, Open Science Framework, Orcid, Phil Papers, SocArXiv, Social Science Research }}$ Network, Vixra and Zenodo websites.

This paper is part of a series on the History of Science and Technology. Other papers in the series are

$\underline{\text { The Invention of Stone Tools } \quad \underline{\text { Fire }} \quad \underline{T h e ~ N e o l i t h i c ~ R e v o l u t i o n ~} \quad \text { The Invention of Pottery }}$

History of Metallurgy The Development of Agriculture and Pastoralism $\quad$ History of Writing

The Invention of Glass $\quad$ History of Astronomy Invention of Microscopes and Telescopes

History of Printing The Invention of the Steam Engine $\quad$ History of Electricity

Electric Telegraph $\underline{\text { Telephone }} \underline{\text { Radio }} \underline{\text { Television }} \underline{\text { Photography }} \underline{\text { Motion Pictures }}$

Internal Combustion Engine Motor Car Aeroplanes The History of Medicine

The Discovery of the Periodic Table The Discovery of the Atomic World

Other papers by Rochelle Forrester include works on Epistemology and the Philosophy of Perception such as Sense Perception and Reality and on quantum mechanics such as the Quantum Measurement Problem, The Problems of Quantum Mechanics and The Bohr and Einstein debate on the meaning of quantum physics. Rochelle Forrester's work is also published on Scribd. Rochelle Forrester is a member of the International Network for Theory of History. 
The change from the traditional Western and Chinese view of the elements involving materials such as water, air, earth, wood, metal and fire, to the chemical elements making up the periodic table, to atoms, to particles such as protons, neutrons and electrons and then to quarks was inevitable. The order of discovery of these ideas of the ultimate constituents of matter was necessary, in that they could not have been discovered in any other order. This was because nature has a particular structure and we have a particular place in nature. The traditional view of the elements could be obtained by naked eye observation, and the view of nature as being made up of the chemical elements in the periodic table was next discovered as it involved the decomposition of the traditional elements such as air and water. This led to the idea there was a separate atom for each element which explained the differences between the elements. The sub atomic particles were discovered in a necessary order with the outer particles like the electron being discovered earlier, and inner particles such as quarks being discovered later. The order of discovery of particles is also affected by the properties of the particles. The charges of particles, their mass and ability to survive outside the particles they make up and other properties will make a particle harder or easier to discover. The order of discovery was inevitable and set by the structure of the universe. The structure of the universe includes the structure of the atom, and of the particles making up the atom, and the properties of the atom, and of the particles making up the atom.

The idea that matter was made up of tiny particles called atoms goes back to Ancient Greece where Leucippus and Democritus claimed all matter consisted of atoms and empty space. This idea was not widely accepted and from the time of Ancient Greece until the late 18th century, the western world generally considered all matter was made up of mixtures, in various proportions, of the basic elements of water, air, earth and fire. Traditional Chinese beliefs were similar and considered all matter was made up of water, earth, wood, metal and fire. The traditional view of the elements ended when scientists showed air was made up of other substances such as nitrogen and oxygen and water was made up of oxygen and hydrogen. The modern view of atomic theory began with John Dalton (1766-1844) who, building upon Lavoisier's idea of chemical elements, suggested that for each chemical element there was a particular atom. He suggested that each element was related to a particular atom with different elements varying from each other due to the particular weight of their atoms. The existence of atoms was widely, but not completely accepted by the late 19th century. It was only with the discovery of the constituents of the atoms and Einstein's explanation of Brownian motion in 1905 that the existence of atoms became completely accepted.

The first of the constituents of the atom to be discovered was the electron. During the 19th century physicists had learned to create and control electric currents. But they had been unable to discover what electricity was as when electricity flows through a wire or gathers on materials that tend to pick up an electric charge, it is impossible to separate the properties of the electricity from those of the wire or other material. In order to understand electricity, it was necessary to separate electricity from the materials that hold the electricity. This meant studying the flow of electricity in a vacuum, or a near vacuum. 
This became possible with the invention of an improved air pump by Johann Geissler in 1858. Geissler's air pump made it possible to remove enough air from a glass tube to create a near vacuum in the tube. Physicists began to create experiments with a glass tube containing a near vacuum with a wire carrying an electric current at one end, known as a cathode, and a positively charged metal plate, known as an anode, at the other end. This apparatus was known as a Crookes tube. When a current was passed through the cathode a thin ray of light passed from the cathode to the anode. The ray of light became known as a cathode ray and was caused by the heating of the small quantity of gas remaining in the tube by something moving through the tube. It was observed that the cathode rays could be deflected by magnets and electric forces placed near the tube which indicated they had an electric charge. In $1897 \mathrm{~J} \mathrm{~J}$ Thompson measured the velocity and the amount of deflection of cathode rays. He found that the velocity of the cathode rays was about a tenth of the speed of light which meant that the cathode rays were not a form of electro-magnetic radiation. The deflection of the cathode rays showed they had a negative electric charge. Given that cathode rays were not electro-magnetic energy the rays had to consist of particles and given that the particles had a negative electric charge they could not be atoms which were normally electrically neutral. Thompson also tried to measure the mass and the charge of the particles but he was only able to measure the ratio of those properties. His experiments showed the ratio remained the same regardless of what gas was in the tube and of what metal was used for the electrode. Eventually in 1909 Robert Millikan was able to separately measure the charge of the electron. This enabled him to calculate the mass of the electron which showed it was a particle vastly smaller than the atom.

Electrons could only come from atoms, and given there was a negatively charged particle within the atom, and that atoms were usually electrically neutral, it seemed obvious that there must also be a positively charged part of the atom. A further question was how the electron and the positively charged material in the atom were arranged. One suggestion by $\mathrm{J} J$ Thompson was that the atom was structured as a plum pudding with positively charged matter making up the great bulk of the atom with negatively charged electrons embedded in the positively charged matter, just as raisins are embedded in a plum pudding. The discovery of the structure of the atom and of the positively charged material in the atom was made by the New Zealand physicist Ernest Rutherford in 1911. Rutherford set up an experiment in which he fired alpha particles at a thin sheet of gold foil and measured the effect on the alpha particles as they were scattered by deflection by the gold foil.

Alpha particles are helium ions, or helium atoms without electrons, so that they are positively charged helium nuclei. Alpha particles had been discovered as one of three types of rays emitted by radioactive elements. The other two rays were beta particles, later discovered to be electrons and gamma rays, a form of electro-magnetic energy of a particular wavelength. Radioactivity had been discovered by the French physicist Becquerel in 1896 and had been extensively studied by Pierre and Marie Curie. The Curies discovered and isolated a radio-active 
element radium which Rutherford used as the source of alpha particles in his experiments when the particles were fired at sheets of gold foil.

Rutherford, assisted by Hans Geiger and Ernest Marsden began a study of the scattering of alpha particles when they were directed at thin metallic foils in 1907. Alpha particles emitted from radium passed through a slit in a lead screen and then hit a metal foil which resulted in some deflection of the alpha particles as they were affected by the atoms of the metal foil. The deflection of the alpha particles was measured when they hit a zinc sulphide screen on which they made a flash of light. It was initially discovered the alpha particles were deflected by no more than a few degrees and that as the angle increased, the number of deflected alpha particles declined. Then Rutherford casually suggested that Geiger and Marsden check to see if any alpha particles were deflected back by a large angle such as more than 90 degrees. To their surprise it was discovered that a few of the alpha particles were deflected backwards by more than 90 degrees. This could not be explained by J J Thompson's plum pudding model of the atom with the positively and negatively charged material spread throughout the atom as widely spread positively charged material could not cause the large angle backward deflection of alpha particles. Rutherford suggested that only the concentration of all the positive charge at a single point in the atom could cause alpha particles to bounce backwards. The few alpha particles which bounced back more than 90 degrees were explained by them bouncing back from the nucleus which occupied a tiny portion of the space of the atom. The positively charged particles making up the nucleus were soon called protons. The great majority of the atom was empty space which explained the large number of alpha particles deflected by a small angle. Those alpha particles simply went through the atom with only a slight deviation from a straight line caused by the positively charged alpha particles being deflected by the positive and negative material in the atom. Measurements were made that showed the nucleus contained nearly all the mass of the atom, but that it occupied only a tiny proportion of the space of the atom.

The discovery of the nucleus of the atom raised questions as to why the negatively charged electrons did not fall into the positively charged nucleus as opposite charges attract each other and why the positively charged nucleus in all atoms with two or more protons (every atom other than hydrogen) did not fly apart as same charged particles repel each other. Neils Bohr suggested that if the electrons were in constant motion around the nucleus in particular orbits then they would not fall into the nucleus due to centrifugal force. Electrons could jump from one orbit to another and could not occupy any position between the orbits. The reason why nuclei of two or more protons do not fly apart is due to the existence of the strong force which holds the nucleus together. The strong force results from protons exchanging special particles known as pions, a process capable of generating an attractive force between protons.

A further problem with the atom was that for atoms other than hydrogen, their atomic weight seemed to be at variance with their atomic number. The atomic number concerns the number of protons and electrons an atom has. Hydrogen has a single proton and electron so its atomic number is 1 and its atomic weight is 1 . Helium, for example, has 2 protons and because it 
is normally electrically neutral, it normally has 2 electrons, so its atomic number is 2 . However the atomic weight of helium is slightly more than 4 and as almost all the weight of an atom is contained in the nucleus its atomic weight would be expected to be two, rather than slightly more than 4 which it had been measured to be. Atoms of the same element had also been discovered by a process known as mass spectrograph to be of different weights. Ions, of the same element, which are atoms which have either lost or gained electrons, so that they are electrically charged were sent through a glass tube containing a vacuum. The stream of ions, being electrically charged, would be deflected by the presence of a magnet and it was observed that the stream of ions would break into several different streams, which suggested the ions had different weights. Lighter ions are deflected more than heavier ions suggesting the reason why the ions stream divided into several different streams was that the original stream consisted of ions of various different weights. Atoms of the same element with different weights became to be called isotopes. The question arose as to why atoms of the same element had different weights. The obvious explanation for these problems was that there was more matter in the nucleus than just the protons. This led Rutherford in 1920 to suggest there must be some as yet undiscovered electrically neutral matter in the nucleus to explain why the atomic weight of atoms, other than hydrogen, were more than double their atomic number and why atoms of the same element could have different weights.

Experiments had been carried out by $\mathrm{W}$ Bothe and $\mathrm{H}$ Becker involving the directing of very fast alpha particles from the radioactive element polonium at various light elements, such as beryllium. It had been observed that the beryllium when struck with the alpha particles emitted unusually highly penetrating radiation. This was initially considered to be electro-magnetic radiation, such as light, but when Irene and Frederick Joliot-Curie noted that the radiation from the beryllium hit a paraffin wax screen it knocked particles out of the paraffin wax at a speed higher than one would expect if the beryllium radiation was electro-magnetic radiation.

James Chadwick suspected the beryllium radiation might be the electrically neutral matter in the atomic nucleus guessed at by Rutherford and which would explain the difference between elements atomic numbers and atomic weights and how different atoms of the same element could be of different weights. In 1932 he investigated the beryllium rays and directed them at a range of materials. Chadwick discovered that, when he directed the rays at materials with increasing atomic weights, the velocity of the particles knocked out of the various materials by the beryllium rays, decreased. The result was consistent with the beryllium rays, being particles with a mass similar to that of protons, rather than electro-magnetic radiation. A further property of the particles were that they were electrically neutral, which was clear due to their considerable penetrating power. Charged particles deflected by electric fields within atoms have much less penetrating power than uncharged particles. The newly discovered electrically neutral particle was initially considered to be made up of a proton and electron, but soon began to be accepted as another elementary particle. The particle came to be called the neutron and in the late 1930 's there were considered to be three elementary particles, the proton, the electron and the 
neutron. The standard model of the atom at that time had the proton and neutron making up the nucleus with electrons orbiting the nucleus in particular orbits. The discovery of the neutron explained why the atomic weight of an atom could be at variance from its atomic number and how different isotopes of an atom could have different weights.

Striking the atomic nucleus with alpha particles had the disadvantage that alpha particles are repelled by the nucleus as both are positively charged. This reduces the power of the collision and limits the target nucleus to the lighter elements, as the heavier elements, containing more protons, repel the alpha particles so strongly that the experiments were ineffective. It occurred to Enrico Fermi, in Rome, that using the newly discovered neutrons could result in more effective bombardment experiments. He systematically began to bombard each element with neutrons, starting with the lightest elements first. When he reached fluorine he discovered that it became radioactive when bombarded with neutrons and that the same effect was observed for many of the heavier elements. The same artificial radioactive effect had been observed by Frederic and Irene Joliot-Curie and was then reproduced by John Cockcroft with his particle accelerator and in America with cyclotrons. It meant that radioactivity which had previously been limited to a few elements could now be produced by many different elements.

Fermi came up with another interesting result when he discovered that hydrogen atoms could slow down neutrons and that slower neutrons generate more activity than faster neutrons. Substances such as water, which contain hydrogen atoms, could slow down or moderate neutron velocities, a discovery that was of importance to nuclear reactors.

Fermi's bombardment of uranium with neutrons and similar experiments carried out by Otto Hahn and Lise Meitner in Berlin, had produced a number of substances which were believed to be new elements but were actually uranium isotopes produced through nuclear fission. Similar experiments, with uranium, carried out by Irene Joliot-Curie and Paule Savitch in Paris produced a substance that looked like the element lanthanum, but which Joliot-Curie and Savitch thought must be actinium, an element close to uranium on the periodic table. Otto Hahn carried out similar experiments and believed he had produced radium, when he had actually produced barium, which chemically was similar to radium.

The reason scientists all over Europe were misinterpreting the results of experiments involving the bombardment of uranium with neutrons was that all the previous bombardment experiments had only knocked a few protons and neutrons out of the nucleus. To turn uranium into a uranium isotope or into actinium which had only three protons less than uranium was no surprise, but to produce barium with only 56 protons or lanthanum with 57 protons to uranium's 92 did not seem possible. Eventually testing of the materials resulting from the bombardment of uranium confirmed the materials were barium and lanthanum and that the bombardment had caused the uranium nucleus to split apart. The results of the experiments were published in early 1939 and the process now called fission was soon being repeated around the world.

It was immediately realised that fission would not only split the nucleus, it would also release additional neutrons which could hit additional uranium nuclei breaking them up to 
release even more neutrons to hit even more uranium nuclei. It was quickly realised that such a process, called a chain reaction would release a tremendous amount of energy, and act either as a bomb or, if appropriately controlled, for peaceful purposes. Scientists had long realised there was enormous energy in the atomic nucleus, but they had no way of releasing it until the development of nuclear fission.

The beginning of the war in Europe in September 1939 sped up research into the possibility of producing an atomic bomb. Scientists in Britain, France and Germany studied how an atomic bomb might be built, although those in Germany showed little enthusiasm for the job. Serious efforts to produce an atomic bomb began in the United States with a letter sent by Albert Einstein to President Roosevelt in September 1939 stating that recent discoveries had made possible the building of a new weapon capable of enormous destruction. The American attempt to build the atomic bomb came to be called the Manhattan Project and was under way by mid-1941 and became increasingly urgent after 7 December 1941 when America entered the war.

Whether a chain reaction was really possible, depended on whether splitting the uranium nucleus would release additional neutrons, and if so, how many. If only a few additional neutrons were released then the chain reaction would gradually reduce and stop as some neutrons are lost during the process. In order to obtain a continual chain reaction it was necessary for the production of additional neutrons to reach what is known as a critical size where the new neutrons being released was equal to the number of neutrons being lost. It had been found that slower neutrons were more effective at breaking up the uranium nucleus, and to slow the neutrons down a moderator such as water or paraffin wax, containing hydrogen atoms was used. It was soon discovered that those substances tend to absorb neutrons making it more difficult to achieve a chain reaction. Eventually it was realised that heavy water or graphite were better moderators as they slowed neutrons without absorbing them. The French were the first to achieve a chain reaction in 1939 but the chain reaction faded without reaching critical size.

A further problem that needed to be sorted out was the separation of the uranium isotopes U-238 and U-235. U-238 was by far the most common form of uranium but U-235 was required for making a bomb. The two isotopes of uranium were usually mixed together with U-235 amounting to only $0.71 \%$ of the mixture. The separation of U-235 from U-238 was achieved by an electro-magnetic separation plant using a two stage process and by a gaseous diffusion plant. Eventually 60 kilograms of U-235 was produced and used in the bomb dropped on Hiroshima.

An alternative to the use of U-235 was the use of Pu-239. This required the building of a nuclear reactor which would turn uranium into $\mathrm{Pu}-239$ which is an isotope of plutonium. The nuclear reactor was built by Fermi with graphite acting as the moderator, with uranium inserted into the graphite reactor in a lattice structure. Fermi's reactor went critical on 2 December 1942 and established a self-sustaining chain reaction. Once the reactor had produced the plutonium it had to be removed from the uranium. Eventually enough plutonium was produced to enable a test bomb to be exploded and for the bomb dropped on Nagasaki. 
Once the U-235 and $\mathrm{Pu}-239$ had been produced it was necessary to turn the material into a bomb. This required some sort of initiator to set the explosion of. It was decided to use the conventional gun method to explode the uranium bomb but this method could not be used for the plutonium bomb. This is because of spontaneous fission caused by the presence of the $\mathrm{Pu}-240$ isotope within the plutonium which could result in pre-detonation. A new method of initiating the explosion, called implosion, was developed for the plutonium bomb. This involved the bomb having a hollow core, surrounded by plutonium which was surrounded by high explosive. The detonation of the high explosive forced the plutonium into the hollow centre of the bomb which creates a supercritical explosive mass. When the bomb is in that state, a burst of neutrons obtained by mixing polonium and beryllium, starts the chain reaction. The implosion method of exploding the bomb was used when the plutonium bomb was tested on 16 July 1945 producing the largest man-made explosion the world had yet seen. Then on 6 August 1945 a uranium bomb was dropped on Hiroshima and on 9 August 1945 a plutonium bomb was dropped on Nagasaki.

The atomic bomb was followed by the hydrogen bomb. The idea of the hydrogen bomb arose among the scientists working on the atomic bomb in the early 1940's. The scientists realised that energy could be produced not just by the fission of heavy nuclei, but also by the fusion of light nuclei, such as hydrogen. Fusion required extreme temperatures such as could be provided by an atomic explosion, so that the atom bomb could provide the heat required for a hydrogen bomb. Work began seriously on the hydrogen bomb and the United States exploded an effective hydrogen bomb in 1954. The Soviet Union exploded an atomic bomb in 1949 and then a small hydrogen bomb in 1953 and a somewhat larger one in 1955. Great Britain soon developed nuclear weapons and was followed by France, China, India, Pakistan and almost certainly Israel. Nuclear weapons proliferation has become a major concern of modern international relations with countries such as Iraq, Libya, North Korea and Iran being suspected of a desire to obtain nuclear weapons. The possibility of terrorist groups obtaining access to nuclear weapons is also of considerable concern.

The discovery of the atomic world did not just lead to the development of weapons of war. Peaceful uses of nuclear fission were developed in nuclear power stations which used nuclear power to create heat, which is used to produce steam, which is used to generate electricity. Nuclear power generated in power stations is made by bombarding uranium with neutrons to produce a chain reaction in the same fashion as atomic bombs work, but the system is controlled by control rods made of materials such as boron, which absorb neutrons. The control rods enable the chain reaction to be started, stopped and to be increased and decreased as desired. The chain reaction produces an extreme amount of heat. The heat is removed from the reactor by pumping heat absorbing materials such as air or water through the reactor. The heat is then used to generate steam which is used to power steam turbines which generate electricity.

Accelerators and nuclear reactors produce various radioactive materials which have important uses in medicine, industry, agriculture and scientific research. In medicine radioactive materials assist diagnosis due to the tendency of some elements to concentrate in particular areas 
of the body. Potassium concentrates in the muscles, iodine in the thyroid and phosphorus in the bones. Cameras can take photos of those organs when a patient is injected with the appropriate radioactive elements. Radioactive elements can be used to treat disease for example radioactive iodine can be used to treat Graves disease, a thyroid disease and thyroid cancer. Radioactive elements cesium-137 and copper-67 are also used to treat cancers. Cobalt-60 is used to sterilize surgical instruments and to treat cancer.

In industry, radioactive substances are used to study the density of materials, to inspect goods for flaws and to track substances passing through piping systems. This is possible because radioactive materials lose energy as they travel through substances and it is possible to detect the energy loss disclosing where the radioactive material is. This is particularly useful where it is not possible to have direct contact with the substance being studied. Radioactive materials are used to check the quality of metal used in cars, to locate and assess oil and mineral deposits and to inspect jet engines and pipelines.

In agriculture radioactive materials have been used in the study of plants and animals. This has produced plants and animals that are more disease resistant and plants that have a greater nutritional value. Improved knowledge of fertiliser use has resulted from the use of radioactive materials to show how plants absorb fertiliser. Radioactive materials have been used to control insect pests such as Mediterranean fruit flies.

Radioactive dating of materials has caused a revolution in both geology and archaeology. In geology radioactive dating has enabled the dating of rocks which has led to calculations of the age of fossils and to rates of evolutionary change and of the age of the earth. Radioactive dating is based on the fact that some isotopes are unstable and decay by the emission of alpha and beta particles and gamma rays. While the rate of decay of an individual isotope is unpredictable the rate of decay of a large quantity of atoms of a particular isotope is predictable. The rate of decay is usually called the isotopes half-life, which is the length of time it takes for half of a given quantity of atoms of a particular isotope to decay. The rate of decay is constant over time so that the quantity of the isotope in some materials will disclose the age of the material. If the half-life of the isotope is one million years and half the isotope has decayed, the material containing the isotope is a million years old.

Radioactive dating is usually done on a mass spectrometer. The mass spectrometer operates by producing a stream of ions from the material which is to be dated. The ions pass through a magnetic field which deflects them based on their mass and charge. This indicates the quantity of the isotope in the material or the degree of decay of the isotope which allows a calculation of the age of the material.

There are a number of unstable isotopes which decay at a predictable rate. U-238 has a half-life of 4.5 billion years, U-235 has a half-life of 700 million years and K-40 has a half-life of 13 billion years. If a given rock has several different unstable isotopes in it, then the dating can be done on both isotopes providing a useful cross check on the dating process. Using radioactive 
dating techniques the age of the earth was calculated in 1953 at 4,550 million years, a figure still accepted today.

Radioactive dating of archaeological samples is usually done using the carbon-14 isotope which is why it is often known as radiocarbon dating. Carbon-14 has a half-life of 5,730 years which makes it useful for dating materials back to around 60,000 years old. Carbon-14 is created by the collision of cosmic rays with nitrogen and oxygen atoms which release neutrons which collide with nitrogen atoms to create carbon-14. The amount of carbon-14 in the atmosphere is very small and the carbon combines with oxygen to form carbon dioxide. Plants absorb the carbon dioxide through photosynthesis and animals acquire the carbon dioxide through eating the plants. When an organism dies, its intake of carbon-14 ceases and the existing carbon-14 in the organism begins to decay at its standard rate. The amount of carbon-14 left in an organism when it is examined indicates how long ago the organism died. If there is plenty of carbon-14, the organism died more recently, if most of the carbon-14 has decayed, then the organism died long ago. A reasonably exact date can be calculated based on the quantity of carbon-14 left in the sample examined. Materials that can be dated include all plant remains and human and animal remains, such as bones. Radiocarbon dating has meant dates have been established for the end of the Neanderthals, the domestication for various animals and plants, the beginning of cities and civilization, the development of writing and many other major prehistoric events.

The only means of studying the atom open to scientists early in the 20th century was through the use of particles, usually alpha particles, generated by natural radiation from radioactive materials such as radium. This method had its limitations due to the limited energy levels of such particles. If the atom could be hit by particles with much greater energy levels a much greater range of effects could be observed. During the 1930's three different methods to deal with this problem were developed. In England John Cockcroft and Ernest Walton developed the voltage doubler accelerator, while in America Robert Van de Graff invented the Van de Graff generator and Ernest Lawrence created the cyclotron. Cockcroft and Walton using their particle accelerator directed a high speed proton beam at lithium and observed alpha particles being emitted from the lithium. This was the first nuclear reaction created by artificial means and its effect was a million times greater than could be produced by alpha particles. However it was the cyclotron that was to become the primary particle accelerator as it could provide a much higher voltage and give much greater energy to particles than the other two machines.

The cyclotron works by continuously sending particles around expanding circular paths and periodically accelerating them to greater and greater speeds. The particles are accelerated by means of electric fields which push and pull charged particles in ever expanding circular paths until the path reaches the borders of the machine. The cyclotron was however limited by accelerating increasingly fast particles in a constant magnetic field. The synchrotron was then developed to apply an increasing magnetic field to the accelerating particles. A synchrotron usually consists of a number of magnets laid out as a hollow ring which accelerates the particles in short bursts by increasing the power of the magnetic field as the particles go by. Synchrotrons 
are usually used for accelerating protons but are unsuitable for accelerating electrons as electrons travelling around a ring will emit photons and lose energy. The solution to this problem was to accelerate electrons in straight lines in linear accelerators. Once these particle accelerators were invented further improvements were made to them for example by using superconducting magnets and designing collider particle accelerators. Superconducting magnets are made from materials capable of being kept at close to absolute zero temperatures and are able to conduct electricity without loss of energy. Collider accelerators involve directing a high energy particle beam, not against a stationary target, but against another accelerated high energy particle beam to create collisions involving much higher energy than there is with a particle beam hitting a stationary target.

A new means of detecting particles, known as the cloud chamber, was invented in 1911 by Charles Wilson and began to be used to detect the results of the collisions of cosmic rays with particles in the atmosphere. Cosmic rays are particles emitted from stars, some of which obtain high energies and penetrate the earth's atmosphere where they will collide with atomic nuclei. The collisions produce a cascade of particles which collide with other nuclei sending even more particles flying round, some of which reach the ground. The cosmic ray collisions result in the production of numerous particles, the presence of which can be observed in cloud chambers.

Cosmic rays were first discovered in 1912 by Victor Hess but were initially believed to be gamma rays and electrons. It wasn't until the early 1930's that cosmic rays were correctly identified as being positively charged particles, mainly protons. When this was understood scientists began to carry out a series of experiments, using cloud chambers, which showed the existence of a range of new particles. The positron was discovered in 1932 by Carl Anderson and in 1937 he and Seth Neddermeyer discovered the muon. The pion, (or pi-meson) was discovered in 1948 and the lambda in 1947. In 1953 the Sigma particle which comes in three types, one positive, one neutral and one negative was discovered. In 1954 the cascade particle, which decays into a lambda and a pion and which comes in two types, one with a negative charge and the other with no charge, was discovered.

Cloud chambers worked by showing the track of a charged particle passing through the chamber, in the form of a line of droplets. The cloud chamber contains a vapour which condenses into droplets around ions produced when the charged particle, passing through the cloud chamber, removes electrons from atoms in the cloud chamber. The cloud chamber can be placed between the poles of a magnet creating an electric field within the cloud chamber. The charged particle will move in a curved path through the cloud chamber, the curve showing whether the charged particle has a positive or negative charge. The degree of curvature and the number of droplets indicates the mass and velocity of the particle.

The cloud chamber was eventually superseded by the bubble chamber in the 1950's. The bubble chamber was much better at detecting high energy particles, such as those produced by particle accelerators, than the cloud chamber. The bubble chamber works in a similar way to the cloud chamber, but is filled with a liquid, usually liquid hydrogen, held under pressure and at a 
temperature just below its boiling point. When particles move through the liquid they create a trail of ions, the pressure on the liquid is then reduced which causes bubbles to form around the ions disclosing the path of the particles. The chamber may be in a magnetic field which discloses the charge of particles and their momentum. The particles are photographed by a number of cameras to show a three dimensional picture of the particle track.

Bubble chambers had some limitations which led to their replacement by the 1980's with more useful particle detectors. Bubble chambers produced photographic rather than electronic results which meant people, rather than computers had to examine the results. This is a problem particularly with experiments that need to be repeated and analysed a number of times. The liquid in the bubble chamber is both detector and target so they cannot be used with accelerators producing particle beam collisions. Bubble chambers lack the size to show the results of high energy collisions and to allow accurate estimates of momentum in such small chambers. These sorts of problems resulted in bubble chambers being superseded by a variety of particle detectors such as spark chambers, wire chambers, drift chambers, silicon detectors, ionisation chambers and other detectors often combined together to produce the best results.

The results of cosmic ray experiments and experiments involving increasingly powerful particle accelerators were the discovery of a vast range of new sub-atomic particles. Many of those particles are very short lived as they quickly decay into other particles but may play important roles in the structure of the atom. By the middle of the 1970's hundreds of particles had been identified and studied. This vast proliferation of particles came to be known as the particle zoo. Attempts were soon being made to classify the particles into various groups based on the properties of the particles. Certain particles such as the electron, muons and neutrino are only involved in the weak interaction, which involves radioactive decay, and are known as leptons. Most other particles, such as protons and neutrons are involved in the strong force that holds the atomic nucleus together and are known as hadrons. Particles which decay into other particles including protons are known as baryons, while those whose decay produces photons (particles of light) are called mesons. Particles which decay slowly are called strange, while those that decay more rapidly are classified as non-strange. Numerical values can be assigned to strange particles. A strange particle decaying into a normal particle, such as a proton, would have a strangeness value of -1 . If it decayed into an antiproton its strangeness value was +1 . If it slowly decayed into another strange particle it had a strangeness value of -2 but if it decayed quickly it had the strangeness value of the particle it had decayed into. Particles may also be classified by angular momentum, which is a property known as spin. Particle spin may be measured in any $1 / 2$ number of whole numbers, for example $1 / 2,1,1 \frac{1}{2}, 2,2 \frac{1}{2}$ and so on. Spin must also be in particular directions such as up, down or sideways. Particles can also be classified by a quality known as isotopic spin. Isotopic spin is based on a particle's electric charge and involves treating particles that are identical, apart from their charge, as being the same type of particles. Particles such as pions, which come with either positive, negative or no charge are treated as the same type of particle but with different isotopic spins. 
The classification of the hundreds of subatomic particles, constituting the particle zoo, made possible a rationalization that came to be known as the eightfold way. Murray Gell-Mann and Yuval Ne'eman independently came up with this rationalization which was similar to the periodic table of the elements. They created a graph with the vertical axis measuring a particles strangeness and the horizontal axis measuring isospin. Particles are represented on the graph by a point. If the eight lightest baryons are put on the graph at points where their strangeness and isospin values intersect the shape produced by the positions on the graph of points representing the baryons strangeness and isospin values forms a hexagon with two particle points being in the centre of the hexagon. The same result is achieved if the eight lightest mesons are positioned on the graph in accordance with their strangeness and isospin values. Why the graphical representation of the particles should produce a hexagon with two particles in the centre was unknown. Gell-Mann later learnt of the mathematics of Lie groups, which was developed in the 19th century. One of the Lie groups called SU(3) dealt with octuplet representations and asserted that the different particles in the octuplets were related in particular ways.

The particle Lie group SU(3) that provided for the octet representation also allowed other representations such as those with ten particles. This was fortunate as not all the groups of particles fitted the octet model. The four delta particles could not fit the octet model but could fit a system of ten particles. The delta particles and five others produced an upside down triangle on the graph with one particle missing at the bottom of the triangle. Gell-Mann using the mathematics of the $\mathrm{SU}(3)$ Lie group predicted the discovery of the new particle and its properties. The new particle was duly discovered in 1963 showing that the SU(3) symmetry worked and was the right way to classify particles.

The question then arose as to why the eightfold way or the SU(3) system worked for the classification of hadrons. The baryons would only form multiplets in accordance with SU(3) mathematics if there was some underlying reason, just as atomic structure provides an underlying reason for the structure of the periodic table. Gell-Mann and George Zweig independently suggested the SU(3) symmetry would make sense if the hadrons were made up of constituents called quarks by Gell-Mann and aces by Zweig. Gell-Mann's terminology was eventually accepted. Quarks were considered to be particles that had electric charges that were only fractions of the charges possessed by electrons and protons. All particles previously identified had electric charges of one (the charges of protons and electrons) or multiples of one. No particle had ever been observed with a charge that was a fraction of one. This was eventually explained by the idea of confinement, whereby quarks, while being the constituents of many particles, could not be knocked free of the particles by accelerator experiments. They cannot be knocked free from particles because as quarks break free from a particle, they form a new particle known as a meson which contains a quark and an antiquark.

The properties of quarks could be deduced as their properties had to explain the properties of the particles they made up. Baryons such as protons and neutrons were made up of three quarks, consisting of two quarks known as up quarks and one down quark for a proton, and 
one up quark and two down quarks for a neutron. Up quarks had an electric charge of $+2 / 3$ and down quarks had an electric charge of $-1 / 3$. Two up and one down quakes for a proton equals $2 / 3$ $+2 / 3+-1 / 3=+1$ being the electric charge of the proton. One up and two down quarks for a neutron equals $2 / 3+-1 / 3+-1 / 3=0$ being the electric charge of the neutron. Leptons, such as electrons, were not made up of quarks at all.

When quarks were first suggested in the early 1960's only the up, down and strange quarks and their antiparticle equivalents were proposed as the constituents of hadrons and mesons. Evidence of the existence of these quarks was discovered in 1967 in experiments where electrons were fired at protons and the electron scattering was measured and suggested protons were made up of other particles. It had been suggested that quarks should come in pairs and there should be an additional quark called the charm quark to partner the strange quark. The J/psi particle was discovered in 1974 and was considered to be made up of a charm quark and its antiquark. The discovery of the charm quark quickly led to general acceptance of the quark model. In 1977 a further quark, the bottom quark was discovered and its partner, the top quark was discovered in 1995.

The quark model had a number of problems that delayed its acceptance. The electron scattering experiments had suggested that the constituent particles of protons were weakly bound and nearly un-interacting within the proton. This suggested it should be easy to knock a quark out of a proton, but no one had been able to do this. The quark model also conflicted with Pauli's exclusion principle that states you cannot have two identical fermions in the same quantum state. Quarks are fermions, yet in some particles, such as the omega minus particle, three identical quarks had the same spin. A further problem was that there needed to be some explanation of why quarks in the same particle with the same charges did not repel each other and destroy the particle they made up.

The solution to these problems was the idea that each quark had to be of one of three colors. Each quark in addition to its other properties had to be either red, blue or green. These colors are not real colors, they are just labels for a particular property quarks possess. The property could just as easily have been called small, medium and large or breakfast, lunch or tea. Each quark is considered to be of one of the three colors so that up quarks for example will be either a red up quark, a blue up quark or a green up quark. Equally every down quark will have color and be either a red down quark, a blue down quark or a green down quark. This applies for all the different types of quarks.

The quark color idea explains why quarks do not exist outside of the particles they make up. This is because the quarks are held together within the particle by the exchange of particles known as gluons. Gluons are able to carry color and create a color field between the quarks when gluons are exchanged. When quakes move apart and threaten to break out of the particle the color field between the quarks becomes stronger and holds the quark within the particle. Should the color field be overcome the quarks will combine to form new particles containing the quarks so that the quarks are never seen independently but are always constituents of a larger particle. 
Quark colors also mean that there is no conflict with Pauli's exclusion principle which states that two identical fermions cannot be in the same quantum state. Quarks in the same particle are not in the same quantum state if they have different colors so the exclusion principle can still apply.

Color has never been detected because the various colors of the quarks, within a particle cancel each other out to make the particle containing the quark colorless. Quarks can only exist in combinations that make the particle they are contained in, colorless.

There are six types or flavours of quarks. These are the up, down, strange, charm, top and bottom quarks. Each quark comes in three colors giving a total of 18 types of quark or 36 if their antiquarks are included. The quarks, plus leptons, such as electrons, constitute the basic constituents of nature. These particles and the various particles involved in the transmission of force, such as gluons, photons and vector bosons constitute the standard model of elementary particles accepted at the current time.

\section{Analysis of the order of discovery of the sub atomic particles and the constituents of matter}

The change from the traditional Western and Chinese view of the elements involving materials such as water, air, earth, wood, metal and fire, to the chemical elements making up the periodic table, to atoms, to particles such as protons, neutrons and electrons and then to quarks was inevitable. The order of discovery of these ideas of the ultimate constituents of matter was necessary, in that they could not have been discovered in any other order. The traditional Western and Chinese views of the elements, was always going to be the earliest explanation of the structure of the material world. This was because the traditional Western and Chinese view of the constituents of matter were based upon elements visible to the unaided senses, so inevitably they were discovered before the chemical elements found in the periodic table most of which are not directly visible to the naked eye and are often mixed up with other substances. It is hardly surprising that pre-scientific societies considered air, water and earth as basic constituents of matter as the air, water and earth are all around us and they seem to represent each of the various forms of matter, gas, liquid and solid. All matter is to some extent like water, if it is liquid, air, if it is a gas and earth, if it is solid. The Chinese elements wood and metal are normally solids and are important to human beings and in the case of wood is an extremely widespread material. Fire is something widespread in human societies and it is of vital importance in a wide range of areas such as cooking, warmth, light, metallurgy and pottery. In general the elements in both the pre-scientific West and China were all around us, reflected the states of matter, solid, liquid and gas and were of great importance to us so it is to be expected that they were considered to be the basic constituents of all matter.

When some of the traditional Western and Chinese elements were broken down, air into a mixture of nitrogen, oxygen and other gases, and water into hydrogen and oxygen, it became clear that the traditional elements were not the basic constituents of matter. The chemical 
elements discovered by scientists in the late 18th and 19th centuries and which became incorporated into the periodic table became the new basic constituents of matter. Dalton's atomic theory was created to explain the differences between the new elements, each element having its own particular atom and being of a different weight from the other atoms. The atomic theory was largely accepted by the end of the 19th century and was confirmed by Einstein's explanation of Brownian motion and the discovery of the constituents of the atom.

The first constituent of the atom to be discovered was the electron. This was because the electron has the property of being easily separated from the atom and because it was the carrier of electricity. Electricity is a force visible to unaided human senses and the electron was discovered through studies made of electricity. Experiments investigating electricity in a near vacuum in a Crookes tube led to the discovery of the electron. Those experiments with electricity in the form of cathode rays showed the cathode rays were made up of particles with an electric charge. Given that the particles were not a form of electro-magnetic radiation due to their low velocity, they could only come from the atoms which constituted matter.

The discovery of the electron was dependent upon the prior discovery of how to control electricity and of how to create vacuums which lead to the creation of instruments such as the Crookes tube. The electron might also have been discovered by investigations of beta decay which consists of electrons or from the bombardment of atoms with alpha particles where the scattering of the alpha particles could have revealed the existence of small negatively charged particles or by the photo-electric effect where electrons are knocked out of metals. Electrons were discovered through the use of Crookes tubes because the Crookes tube was the earliest method for the discovery of electrons to become available. However whatever method was used it is quite clear that the electron was only going to be discovered in the late 19th or early 20th century. It was not going to be discovered by the Romans or in Medieval Europe and Asia. It could only be discovered after certain prior discoveries had been made and those discoveries had not been made during Roman and Medieval times.

The proton was the next particle discovered because it was a charged particle and the atom required a positively charged particle to balance the negatively charged electron, so that the atom could be electrically neutral. The discovery of the proton and the atomic nucleus was made by Rutherford's bombardment of gold foil by alpha particles and the analysis of the alpha particle scattering. This was only possible because of the prior discovery of radioactivity by Becquerel in 1896 and alpha particles which quickly followed from Becquerel's discovery. Rutherford's experiment was only possible because nature had provided radioactivity, a form of decay of unstable elements, which involves the emission of alpha particles which could be used to bombard other substances. If radioactivity and alpha particles did not exist, protons would not have been discovered when they were. Their discovery would have been delayed until either the development of particle accelerators, which first occurred in the 1930's or cosmic ray experiments which also began in the 1930's. 
The neutron was discovered in 1932 and was the last of the constituents of the atom to be discovered. The discovery occurred later than that of the other atomic constituents due to the particular properties of the neutron. Unlike the proton and the electron the neutron carries no electric charge, so its existence will not be disclosed by experiments involving the scattering of charged particles fired at the atomic nucleus. If the neutron had a charge it would have an effect on particles fired at the atomic nucleus, either attracting them or repelling them and so showing its existence. As it has no charge, it has no effect on such particles, so it was quite hard to detect the neutron. The neutron, unlike the proton and the electron does not survive outside the atomic nucleus, as when it is outside the nucleus it decays into a proton, an electron and an antineutrino. The neutron has an important property, its mass and this property was what allowed its discovery. The neutron's mass explained why the elements other than hydrogen had atomic weights in excess of their atomic numbers and why different isotopes of the same atom could exist. It was the mass of the neutron that enabled the neutron to knock particles out of the paraffin wax in experiments involving beryllium rays. It was the properties of the neutron, such as its lack of charge and inability to survive outside the nucleus that were the reasons for the neutron being the last constituent of the atom to be discovered. It was however its property of having mass that led to its discovery. In general it was the properties of the constituents of the atom that determined their order of discovery. The particles with charges and which survive outside the atom were discovered before the particle with no charge and which decays into other particles when outside the atom.

The simple system of the proton, neutron and electron as the basic constituents of matter was not to last. The invention of a series of new instruments such as particle accelerators and particle detection methods such as cloud chambers, bubble chambers and other detectors were to result in the discovery of a vast range of new particles. The particles were to be discovered using both particle accelerator and cosmic ray experiments. These particles which were to collectively be called the particle zoo could not have been discovered without the invention of particle accelerators or without the existence of cosmic rays. If nature did not provide cosmic rays, or if particle accelerators could not be built, for example if it was not possible to accelerate particles due to the electro-magnetic force not existing, then it most likely would not have been possible to discover those particles. If it was not possible to detect particles by means of instruments such as cloud and bubble chambers we would not have known of the existence of many of the particles that make up the particle zoo. If nature had been different then the particle zoo may never have been discovered so human history would have been different.

The particle zoo inevitably led to a study of those particles and that led to a classification scheme which resulted in the eightfold way analysis of the particles independently developed by Murray Gell-Mann and Yuval Neeman. The eightfold way which was based on SU(3) Lie group mathematics revealed a pattern among the particles which indicated some underlying situation that gave rise to the pattern. Using the pattern provided by the eightfold way Murray Gell-Mann and George Zeweig were able to predict that hadrons, which include protons and neutrons, were 
made up of particles that were eventually called quarks. Acceptance of the quark model took some time but eventually the discovery of quarks and the idea of colors which explained why quarks did not exist outside of hadrons and why quarks did not contradict Pauli's exclusion principle lead to the acceptance of the quark model.

The idea of quarks could only be developed after the discovery of the particle zoo. It was the particles making up the particle zoo, and more particularly their properties that provided Gell-Mann and Neeman with the information that led to their suggestion that hadrons were made up of particles that were to be called quarks. The existence of these particles and their properties could explain the properties of the particles they made up. This idea is based upon various graphs representing particles and showing a pattern among the particles that could be explained by some underlying factor such as hadrons being made up of other particles which are now called quarks. The properties of quarks such as charge, spin and isotopic spin could be calculated from the properties of the hadrons they made up. This meant that quarks could not be conceived of until after the discovery of the particle zoo and the eightfold way analysis of the particle zoo.

There is a hierarchy in the discovery of the constituents of matter from the original Western and Chinese traditional elements, to the chemical elements that make up the periodic table, to atoms, to protons, neutrons and electrons, which are the constituents of atoms, to quarks which are the constituents of protons and neutrons. Human ideas of the constituents of matter had to follow that order of discovery, with each particle being discovered before its constituents, as it was the order of discovery of that which is closest to us to that which is furthest from us. The order of discovery of particles is also affected by the properties of the particles. The charges of particles, their mass and ability to survive outside the particles they make up and other properties will make a particle harder or easier to discover.

Nuclear weapons are only possible due to nuclear fission. Nuclear fission is only possible due to the existence of very heavy elements such as uranium and plutonium and to the existence of the neutron. If the very heavy elements like uranium and plutonium did not exist then fission would not be possible as it is only those elements that will break apart when hit by neutrons and release their own neutrons to start a chain reaction. Only very heavy elements contain sufficient neutrons to create a sustainable nuclear reaction.

The second requirement for nuclear fission is the existence of neutrons. If neutrons did not exist then nuclear fission and nuclear bombs and power would not exist. Neutrons are used to start the fission process, but if neutrons did not exist then possibly some other particles could be used for that purpose. However for the continuation of the process, the creation of a chain reaction neutrons are necessary as the only other constituent of the nucleus, the proton would be repelled by other uranium or plutonium nuclei as they are both positively charged. If nature did not provide both heavy elements such as uranium and plutonium or did not provide the neutron then nuclear fission would not be possible. If nuclear fission was not possible then both nuclear bombs and nuclear power would not be possible. 
Nuclear bombs and power could not be invented until after uranium and plutonium had been discovered. Uranium was first discovered in 1789 and was isolated in 1841. Plutonium was first produced in a cyclotron in 1941. The important discovery was that of the neutron by James Chadwick in 1932. As soon as the neutron had been discovered scientists began to use it to bombard various elements as its lack of an electrical charge made it a more effective bombardment tool than the positively charged alpha particles. The bombardment of uranium resulted in nuclear fission and the awareness among physicists that the breaking up of the uranium nucleus would release enormous amounts of energy that could be turned into a bomb. The Second World War caused research into the making of a nuclear bomb to be sped up so that both plutonium and uranium bombs were produced by 1945. If there was no Second World War nuclear weapons would have taken somewhat longer to develop, but even without the war nuclear weapons would have been created probably in the 1950's.

The development of nuclear weapons led to the development of nuclear power when the world's first nuclear reactor was built in 1942 and created the world's first self-sustaining chain reaction. Nuclear reactors for the generation of electricity began to be built in the 1950's and 1960 's in many countries.

Nuclear weapons and nuclear power are only possible because nature provides the materials for nuclear weapons and power in the form of heavy elements such as uranium and allows the production of plutonium and because nature provides neutrons which allow the creation of a sustained chain reaction within reactors. Without uranium and plutonium and neutrons history would be greatly different as there would have been no nuclear weapons or power in the 20th century. Given that nature provided uranium and neutrons and allows the production of plutonium, human curiosity about the world around us, would inevitably, sooner or later have led to the development of nuclear weapons and power. Nuclear weapons and power were developed, when they were in the 20th century as they could not have been developed until a vast number of prior discoveries had been made.

The use of radioactive materials in medicine, industry, agriculture and science only occurred after the discovery of radioactivity and knowledge of how it could be created in accelerators and nuclear reactors and the realisation that such materials could be useful in medicine, industry, agriculture and science. Radioactivity was discovered in 1896 and the realisation that it could be created in cyclotrons and reactors occurred quickly after the invention of those machines. Studies of radioactive materials lead to the realisation of their uses in medicine, industry, agriculture and science. In particular, their use in radioactive dating, led to a revolution in ideas of the age of the earth and in the dating of prehistoric and historic organic materials. Previous ideas of the age of the earth and of organic remains had no objective basis and were just unscientific guesses. Only with the advent of radioactive dating were reliable dates able to be established in archaeology and geology.

It is only because nature provides radioactivity that we have radioactive dating. If nature did not provide radioactivity we would still have no real idea of the age of the earth or of 
archaeological remains. Nature also allows the creation of radioactive materials in cyclotrons and nuclear reactors which provide materials for medical, industrial, agricultural and scientific uses. If nature did not allow this artificial creation of radioactive materials, their use in medicine, industry, agriculture and science would be vastly reduced. We would only be able to use naturally occurring radioactive materials and if nature did not provide them we would have no sources of radioactive materials for use in medicine, industry, agriculture and science.

The analysis of the discovery of the atomic world and the constituents of matter shows how the discovery proceeded through a particular path and that it had to proceed through that path. Particles were discovered in a logical order and when they were discovered the social and cultural effects of the discovery appeared in human history. When the neutron was discovered and combined with heavy elements such as uranium and plutonium nuclear weapons and power were quickly invented. The discovery of radiation and the artificial creation of radiation in accelerators and nuclear reactors were quickly followed by the use of radioactive materials in medicine, industry, agriculture and science and for radioactive dating. If however the structure of matter was different, for example there were no neutrons or radioactivity, there would have been no nuclear weapons or power and no use of radioactivity in medicine, industry, agriculture and science and for the dating of ancient materials. If however the structure of matter was different it may have provided humans with other means for meeting human needs. If nuclear fusion was possible at normal temperatures we may have had an abundant, clean and safe energy source which may have ended human reliance on fossil fuels and the concerns about the greenhouse effect. If the structure of matter was different then certainly the course of human social and cultural history would have been different.

\section{Bibliography}

Davies, P C W (1979) The Forces of Nature, Cambridge University Press, Cambridge

Kane, Gordon (1995) The Particle Garden: Our Universe as Understood by Particle Physicists, Addison-Wesley Publishing Co, Reading, Massachusetts

McKay, Alwyn (1984) The Making of the Atomic Age, Oxford University Press, Oxford, New York

Oerter, Robert (2006) The Theory of Almost Everything, Pi Press, New York

Squires, Euan (1985) To Acknowledge the Wonder, Adam Hilger Ltd, Bristol and Boston

Trefil, James (1994) From Atoms to Quarks, An Introduction to the Strange World of Particle Physics, Doubleday, New York

Weinberg, Steven (2003) The Discovery of Subatomic Particles, Cambridge University Press, Cambridge 